\title{
Síndrome de Mowat-Wilson: caso clínico
}

\author{
VANIA A. VILLOTA D. ${ }^{1}$, WILMAR SALDARRIAGA G. ${ }^{2}$, JUAN FERNANDO GÓMEZ C. ${ }^{3}$ \\ 1. Médico Interno, Investigación Genética de Trabajo Estudiantil, Escuela de Medicina, Facultad de Salud, \\ Universidad del Valle, Cali, Colombia. \\ 2. Profesor Asociado, Departamentos de Morfología y Gineco-Obstetricia. Facultad de Salud, Universidad del Valle, \\ Especialista Ginecología y Obstetricia. Maestría en Ciencias Básicas Médicas con énfasis en Embriología y Genética. \\ Grupo de Malformaciones Congénitas Perinatales y Dismorfología. MACOS. Cali, Colombia. \\ 3. MD., MSC. Pediatra, Neurólogo pediatra; Profesor Asistente, Departamento de Medicina Materno-Infantil, \\ Universidad ICESI; Fundación Valle del Lili. Colombia.
}

\begin{abstract}
Mowat-Wilson's syndrome: a case report

Introduction: Mowat-Wilson syndrome (MWS) is a very infrequent congenital polimalformative disorder, caused by mutations, deletions or insertions of the ZEB2 gene, which codify for a protein that is involved in the migration of the neural crest cells and the development of middle line structures. The inheritance pattern is autosomal dominant, haplo-insufficient. Objectives: To describe a case of Mowat-Wilson syndrome with typical phenotype. Clinical case: 8 year-old female, with special facie, hard to control seizures, ductus arteriosus, left pulmonary artery stenosis, constipation, severe retardation of psychomotor development and speech retardation. Differential diagnosis of Mowat Wilson syndrome is proposed. Conclusion: Mowat and Wilson syndrome is very rare not well-known; therefore it is important to communicate its features within the pediatric community. In this syndrome multiple organs and systems are involved, so a multidisciplinary approach is mandatory; in order to the objective of adequately intervening in the pathology of each patient, provide adequate information regarding the developmental expectations for the child and genetic and reproductive counseling to the parents.
\end{abstract}

(Key words: Mowat-Wilson's syndrome, Hirschsprung's disease, congenital defects, epilepsy, congenital heart defects, dominant, haplo-insufficient).

Rev Chil Pediatr 2012; 83 (4): 371-376

\section{RESUMEN}

Introducción: El síndrome de Mowat-Wilson (MWS) es un síndrome polimalformativo congénito de muy baja frecuencia causado por mutaciones, inserciones o deleciones del gen ZEB2, que codifica para una proteína que está involucrada en la migración de las células de la cresta neural y en el desarrollo de las estructuras de la línea media. El patrón de herencia es autosómico dominante, haploinsuficiente. Objetivo: Describir un caso con fenotipo característico de Síndrome de Mowat-Wilson. Caso clínico: Paciente femenino de 8 años, con facies especiales, síndrome convulsivo de difícil control, ductus arterioso, estenosis de la rama izquierda de la arteria pulmonar, estreñimiento, retraso severo del desarrollo psicomotor y del lenguaje en el cual se plantea el

Trabajo recibido el 27 de abril de 2011, devuelto para corregir el 01 de agosto de 2011, segunda versión el 31 de enero de 2012, aceptado para publicación el 23 de abril de 2012.

Correspondencia a:

Wilmar Saldarriaga

E-mail:wsaldarriaga0608@yahoo.com. 
diagnóstico clínico de síndrome de Mowat-Wilson. Conclusión: El Síndrome de Mowat Wilson es un síndrome de baja frecuencia, poco conocido, por lo que es importante difundir sus características en la comunidad pediátrica. En este síndrome se afectan múltiples sistemas y órganos, lo cual requiere un manejo multidisciplinario con el objetivo de intervenir adecuadamente en la patología de cada paciente, dar adecuada información de las expectativas del desarrollo del niño y consejería genética y reproductiva la familia.

(Palabras clave: Síndrome de Mowat-Wilson, enfermedad de Hirschsprung, defectos congénitos, epilepsia, defectos cardíacos congénitos, autosómico dominante, haploinsuficiente).

Rev Chil Pediatr 2012; 83 (4): 371-376

\section{Introducción}

El síndrome de Mowat-Wilson (SMW) (OMIM \#235730) es un trastorno del desarrollo poco frecuente, descrito por primera vez en 1998 por Mowat et al, caracterizado por retraso mental, epilepsia, facie típica que incluye escaso cabello, frente amplia, puente nasal deprimido, con punta de nariz prominente, orejas rotadas posteriormente con lóbulo prominente hacia arriba; filtro nasolabial con surco profundo, labio superior en forma de "M", labio inferior grueso y/o evertido, mentón triangular; y un amplio espectro de características clínicas heterogéneas sugerentes de neurocristopatías a nivel cefálico, cardíaco y vagal. Hasta el momento no hay datos sobre la prevalencia de este síndrome y sólo existen reportes de casos, sin embargo, se sabe que presenta un patrón de herencia autosómico dominante causado por mutaciones heterocigotas dominantes o por deleciones en el gen de los dedos de zinc de la caja E vinculantes con la caja homeótica ZEB2, también reportado como ZFHX1B o SIP1 $1^{1}$.

Se reporta un caso de paciente con epilepsia de muy difícil control, que fue manejado por neurólogo y genetista, logrando asociar múltiples características clínicas llegando al diagnóstico clínico de síndrome de MowatWilson.

\section{Objetivo}

Comunicar un síndrome poco conocido en la comunidad pediátrica y poner énfasis en el aporte que pueden dar grupos interdiciplinarios para lograr diagnósticos sindromáticos en pacientes con múltiples defectos congénitos, de origen genético, de muy rara ocurrencia y difícil acceso a pruebas moleculares, como es el SMW.

\section{Caso clínico}

Paciente de 8 años de edad, sexo femenino, evaluada por neurología pediátrica en una institución nivel IV en la ciudad de Cali, Colombia. Hija de padres no consanguíneos, sin antecedente familiar de casos de retardo mental, cardiopatía, epilepsia o enfermedad de Hirschsprung. Producto de primer embarazo, madre de 25 años al momento del parto, con un control prenatal apropiado, con talla, peso y perímetro cefálico adecuados para la edad gestacional. Desde los 6 meses notan pobre desarrollo psicomotor y del lenguaje, deterioro progresivo en los percentiles de talla, peso y perímetro cefálico. Presentó infecciones del tracto urinario a repetición con ecografía renal y de vías urinarias normal; estreñimiento hasta de 7 días con enema baritado que mostró dilatación generalizada de todos los segmentos del colon, sin evidencia de zona de transición, sugiriendo síndrome de retención fecal funcional, que se manejó médicamente. Síndrome convulsivo de difícil manejo desde los 5 años, evaluada por neurólogo concluye síndrome convulsivo tónico clónico focal tratado con ácido valproico, fenobarbital, carbamazepina y fenitoína con mala respuesta. $\mathrm{Al}$ examen físico se observaba un retraso en el desarrollo psicomotor caracterizado por marcha con tropiezos, necesidad de ayuda para subir escaleras; no controlaba esfínteres, requería ayuda para necesidades básicas tales como comer y vestirse; pronunciaba monosílados y presentaba un comportamiento autoagresivo con automutilación y tricotilomanía. 
En la evaluación por genética destacaba: $16 \mathrm{~kg}$ de peso (percentil < 2), 114 $\mathrm{cm}$ de talla (percentil $<2$ ), perímetro cefálico $46 \mathrm{~cm}$ (percentil <0,5). Facies dismórficas caracterizada por frente prominente, cejas ensanchadas, muy pobladas medialmente y lateralmente escasas; telecanto, ojos hundidos, fisuras orbitarias inclinadas hacia abajo; nariz con punta prominente y columnela larga; orejas rotadas hacia atrás con el lóbulo grande y plegado hacia arriba, hélix redundante (figura 1). Extremidades atróficas, marcha con aumento de la base de sustentación (figura 2). Cariotipo con bandas $\mathrm{G}$ reportó 46 , XX, descartando alteraciones cromosómicas numéricas y estructurales observables al nivel de resolución 550 bandas. Tomografía axial computarizada y resonancia magnética nuclear mostraron atrofia cortical progresiva (figura 3). Ecocardiograma realizado a los 8 años de edad mostró ductus arterioso persistente amplio, estenosis severa de la rama izquierda de la

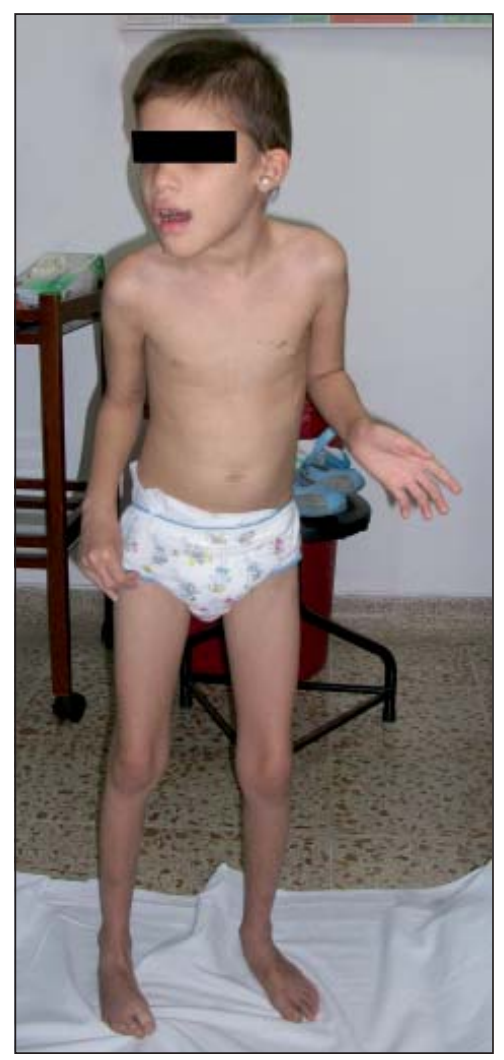

Figura 2. Fotografía de la paciente con SMW de 8 años. Nótese extremidades atróficas, disminución de la masa muscular, aumento de la base de sustentación.

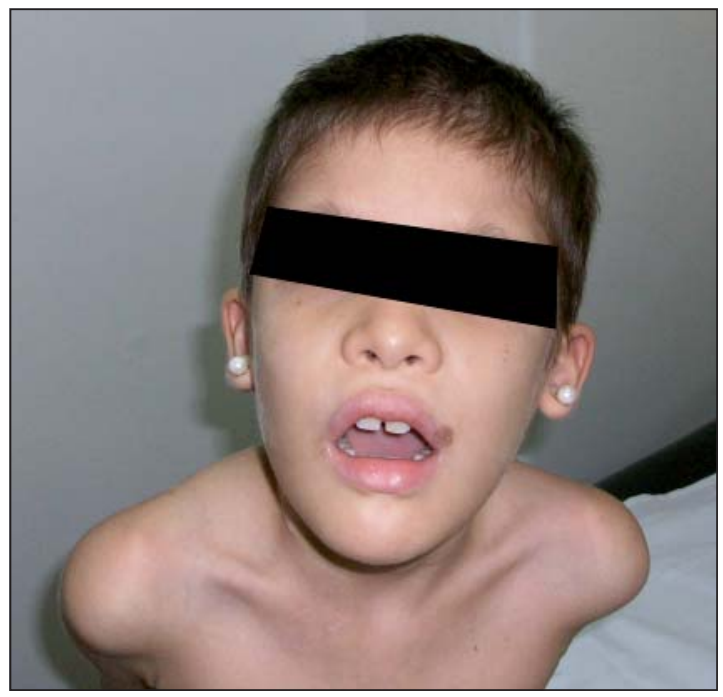

Figura 1. Fotografía de la paciente con SMW. Acercamiento a la cara. Se observa: Facies dismorficas: frente prominente; cejas ensanchadas, muy pobladas medialmente y lateralmente escasas; telecanto, ojos hundidos, fisuras orbitarias inclinadas hacia abajo; nariz con punta prominente y columnela larga; orejas rotadas hacia atrás con el lóbulo grande y plegado hacia arriba, hélix redundante.

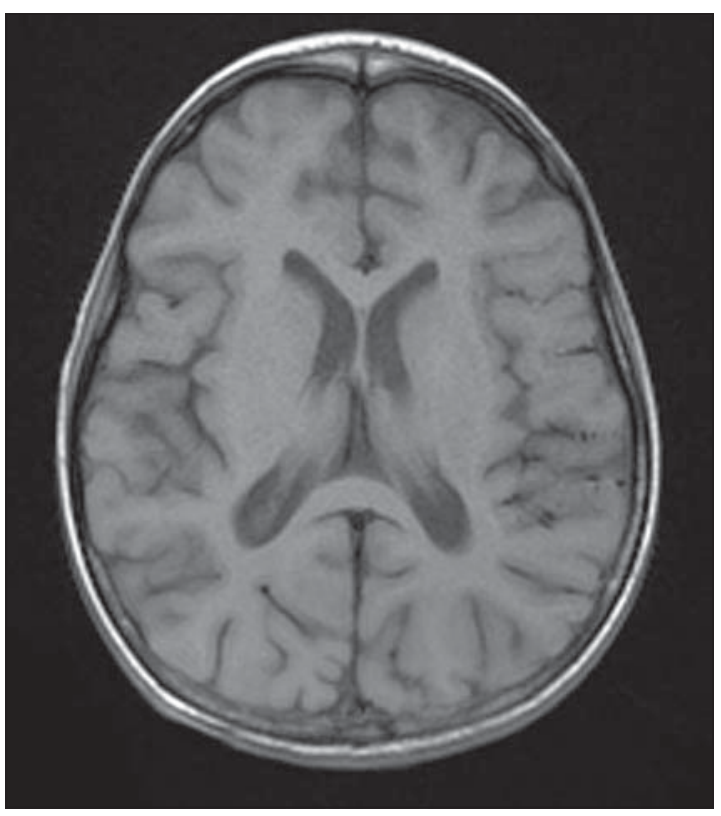

Figura 3. Imagen por Resonancia Magnética potenciada en T1. Corte axial a nivel de la región gangliobasal, se observa prominencia del espacio subaracnoideo en relación a atrofia cortical. Se observa indemne el cuerpo calloso. 
arteria pulmonar e hipertensión pulmonar severa. En la evaluación oftalmológica se encontró atrofia del nervio óptico sin otros hallazgos.

Dados los antecedentes médicos y las características clínicas y laboratorio se hizo el diagnóstico de Sd. Mowat Wilson.

\section{Discusión}

La prevalencia del síndrome de Mowat Wilson (SMW), es desconocida actualmente, pero es probable que haya un subregistro muy importante, especialmente en pacientes con enfermedad Hirschsprung (HSCR) ${ }^{2}$. Garavelli y Cerruti-Mainardi en el 2007, afirman que hasta el momento de esa publicación, desde la primera descripción por Mowat et al (1998), se habían reportado aproximadamente 171 pacientes con las características clínicas del SMW con alteraciones en el gen ZEB2. Estas han sido reportadas principalmente en el norte de Europa, Australia, Italia y los Estados Unidos. La razón hombre/mujer es de aproximadamente 1,42:1, el síndrome se ha identificado en varios grupos étnicos, con características clínicas similares en todas las poblaciones ${ }^{3}$.

Este síndrome es una enfermedad genética autosómica dominante causada por mutaciones genéticas puntuales o deleciones en el gen de los dedos de zinc de la caja $E$ vinculantes con la caja homeótica (ZEB2), situado en el locus 2q21-21q23, el cual es un corepresor transcripcional que juega un papel importante en la migración de las células de la cresta neural y en el desarrollo de las estructuras situadas en la línea media tales como el sistema gastrointestinal y el corazón ${ }^{4}$.

Los primeros aspectos clínicos fueron descritos por Mowat et al en 1998, quien describió 6 casos aislados de niños que tenían en común un fenotipo facial distintivo asociado a retraso mental, microcefalia y baja talla. Además, 5 de ellos presentaban HSCR, de los cuales 4 estaban afectados desde el nacimiento ${ }^{5}$.

Los rasgos faciales característicos son frente alta y abombada, cejas grandes con ensanchamiento medial y adelgazamiento lateral, hipertelorismo, ojos grandes y profundos, orejas rotadas hacia atrás, lóbulos grandes e im- plantados anteriormente, nariz y puente nasal prominente con la punta nasal redondeada y columella prominente, boca persistentemente abierta, labio superior en forma de "M", mentón estrecho triangular y puntiagudo. Estos hallazgos se acompañan de un compromiso del desarrollo psicomotor y del lenguaje, epilepsia de difícil manejo y otras menos frecuentes, entre ellas la enfermedad de Hirschsprung, constipación, anomalías genitourinarias como hipospadias en hombres, cardiopatías congénitas siendo el ductus arterioso persistente la más frecuente, agenesia del cuerpo calloso y oculopatías. Se debe tener en cuenta que el fenotipo cambia dependiendo de la edad, acentuándose los rasgos faciales, generalmente asociados a un retraso mental severo ${ }^{1-5}$. Todas estas características faciales las tenia el paciente aquí reportado, además presentaba retraso del desarrollo psicomotor y del lenguaje, epilepsia de difícil control, constipación, ductus arterioso persistente y atrofia del nervio óptico, con lo cual se sustenta clínicamente el diagnóstico de síndrome de Mowat Wilson. HSCR no fue descartado, dado que el estreñimiento tuvo manejo medico y no se le realizo manometría anal ni biopsia de tejido rectal.

El diagnóstico diferencial de SMW se hace con otros síndromes que se caracterizan por retardo del desarrollo psicomotor y del lenguaje severo, convulsiones de difícil control, enfermedad de Hirschsprung, cardiopatía, talla baja, facies dismorficas. Incluye síndrome de Angelman, del cual la paciente no presentaba las facies específicas, la personalidad afectuosa, y en este síndrome el cuadro convulsivo disminuye la intensidad con el paso del tiempo, contrario a la paciente en la era cada vez de más difícil control. Otro síndrome a diferenciar es Goldberg Shprintzen (GOSHS), el cual comparte los hallazgos clínicos de HSCR (diagnóstico no concluyente en nuestra paciente), epilepsia y retardo mental; se diferencian en los hallazgos faciales, en GOSHS se observa puente nasal alto, sinofris, pestañas largas rizadas, ptosis palpebral, y paladar hendido ${ }^{3}$, que la paciente no tenía.

Hasta el momento, se han reportado más de 110 deleciones o mutaciones en pacientes con un fenotipo típico. Generalmente, son de- 
leciones completas o mutaciones de parada, lo que sugiere que el principal mecanismo es la haploinsuficiencia ${ }^{6}$. Estudios de análisis de genotipo-fenotipo muestran que la severidad del cuadro depende de compromiso del gen mutado, por lo tanto, las mutaciones que codifican para un codon de parada y las deleciones extensas van a tener un fenotipo más pronunciado. Las características faciales y el retraso en el desarrollo psicomotor siempre están presentes, mientras que el resto de malformaciones congénitas pueden variar. Un pequeño número de pacientes presentan un fenotipo atípico debido a mutaciones inusuales. La mayoría de los casos notificados hasta el momento son esporádicos, por lo tanto, el riesgo de recurrencia es bajo en padres sanos. Sin embargo, algunos casos excepcionales se han reportado hermanos afectados, posiblemente por un mosaicismo paternal o en la línea germinal ${ }^{7}$.

Las características clínicas en el síndrome de Mowat-Wilson son muy particulares y el diagnóstico se sustenta en ellas, dado que el análisis molecular de ZEB2 no es de fácil acceso desde Latinoamérica y en Estados Unidos y Europa tiene altos costos. La paciente aquí reportada presenta las características clínicas clásicas de MWS (tabla 1).

El manejo para el síndrome de Mowat-Wilson debe ser individualizado según el grado de severidad. Para ello, siempre que se sospeche un caso, este debe ser valorado por un genetista que identifique las características relacionadas, y de ser necesario, descartar otras anomalías congénitas que se presentan en el síndrome y que, al momento del examen no hayan sido exploradas, además de descartar alteraciones cromosómicas numéricas y estructurales a través de un cariotipo. En este caso, la paciente presenta un cuadro convulsivo desde los 5 años, por lo que requiere un manejo oportuno por neurología pediátrica. Igualmente, los hallazgos del ecocardiograma necesitan la valoración clínica de un cardiólogo. Concomitantemente es menester iniciar terapia ocupacional, física y del lenguaje para ayudar a desarrollar nuevas formas de comunicación, ya que la mayoría de pacientes que padecen este síndrome no aprenden a hablar. Por otro lado, el apoyo sicológico a los familiares es
Tabla 1. Características fenotípicas asociadas al síndrome de Mowat-Wilson ${ }^{3}$

\begin{tabular}{|lc|}
\hline Reportadas por la literatura & $\begin{array}{c}\text { Presentes en } \\
\text { el paciente }\end{array}$ \\
\hline $\begin{array}{l}\text { Retraso del desarrollo psicomotor y del } \\
\text { lenguaje (99\%) }\end{array}$ & + \\
\hline Facies características (97\%) & + \\
\hline Microcefalia (81\%) & + \\
Convulsiones (73\%) & + \\
\hline HSCR (57\%) & No hay reporte \\
Cardiopatías congénitas (52\%) & + \\
\hline Baja Talla (46\%) & + \\
\hline $\begin{array}{l}\text { Agenesia o hipoplasia del cuerpo calloso } \\
\text { (43\%) }\end{array}$ & + \\
Constipación (26\%) & + \\
Anomalía renal (12.8\%) & - \\
\hline
\end{tabular}

Anomalías más frecuentes en el SMW. Los hallazgos positivos en la pacientes llevan un "+" y los que están ausentes un "-"

fundamental para garantizar una mejor calidad de vida, tanto para la paciente como para sus padres. Idealmente comunicar a la familia con fundaciones relacionadas.

\section{Conclusión}

El síndrome de Mowat Wilson es un síndrome de baja frecuencia, poco conocido, por lo que es importante difundir sus características en la comunidad pediátrica; haciendo énfasis en que en este síndrome se afectan múltiples sistemas y órganos, lo cual requiere un manejo multidisciplinario con el objetivo de intervenir adecuadamente en la patología de cada paciente, dar adecuada información de las expectativas del desarrollo del niño y consejería genética y reproductiva la familia.

\section{Consideraciones éticas}

Los datos de la historia clínica y las fotografías de la paciente fueron tomados y publicados con consentimiento informado firmado por la madre de la paciente. Los autores declaran que no hay conflicto de intereses en el presente manuscrito. 
VILLOTA V. y cols.

\section{Referencias}

1.- Online Mendelian Inheritance in Man: Mowat-Wilson syndrome. Baltimore: Johns Hopkins University; 1988 [Actualizado 07/01/2009]. Disponible en: http://www. ncbi.nlm.nih.gov/omim/235730.

2.- Cerruti-Mainardi P, Garavelli L, Pastore G, et al: Mowat-Wilson syndrome and mutation in the Zinc Finger Homeo Box 1B Gene: a new syndrome probably under-diagnosed. Italian J Pediatr 2005; 31: 116-25.

3.- Garavelli G, Cerruti-Mainardi P: Mowat-Wilson Syndrome. Orphanet Journal of Rare Diseases 2007; 2: 42.

4.- Zweier C, Albrecht B, Mitulla B, et al: "Mowat-Wilson" Syndrome with and without Hirschsprung Disease is a distinct, recognizable Multiple Congenital Anomalies-
Mental Retardation Syndrome caused by Mutations in the Zinc finger homeobox $1 \mathrm{~B}$ gene (ZFHX1B). Am J Med Genet 2002; 108 (3): 177-81.

5.- $\quad$ Mowat DR, Croaker GDH, Cass DT, et al: Hirschsprung disease, microcephaly, mental retardation, and characteristic facial features: delineation of a new syndrome and identification of a locus at chromosome 2q22-q23. J Med Genet 1998; 35: 617-23.

6.- Cecconi M, Forzano F, Garavelli L, et al: Recurrence of Mowat-Wilson syndrome in sibling with a novel mutation in the ZEB2 gene. Am J Med Genet A 2008; 146A (23): 3095-9.

7.- Zweier C, Thiel CT, Dufke A, et al: Clinical and Mutational Spectrum of Mowat-Wilson Sindrome. Eur J Med Genet 2005; 48: 97-111. 